\title{
Mitochondrial activity of cancer and normal mesenchymal stem cells in vitro cultured in medium with different deuterium content
}

\author{
Igor Zlatskiy ${ }^{1,2, *}$, Nadine Antipova ${ }^{1,3}$, Alona Zlatska ${ }^{2,4}$, Svitlana Dolenko ${ }^{5}$, and Anton \\ Syroeshkin ${ }^{1}$ \\ ${ }^{1}$ Peoples Friendship University of Russia (RUDN University), 117198, Miklukho-Maklaya Str., 6, \\ Moscow, Russia \\ ${ }^{2}$ State Institute of Genetic and Regenerative Medicine of NAMS of Ukraine, 04114, Vyshhorodska \\ Str., 67, Kiev, Ukraine \\ ${ }^{3}$ Shemyakin-Ovchinnikov Institute of Bioorganic Chemistry, RAS, 117997, Miklukho-Maklaya Str., \\ 16/10, Moscow, Russia \\ ${ }^{4}$ Biotechnology Laboratory ilaya regeneration, medical company ilaya, 03115, Kiev, Ukraine \\ ${ }^{5}$ Institute of Colloid and Water Chemistry, NAS of Ukraine, 03142, Akademika Vernadskoho \\ Boulevard, 42, Kyiv, Ukraine
}

\begin{abstract}
We showed that cancer and normal mesenchymal stem/stromal (MSC) cells in vitro in a deuterated growth medium show a decrease of mitochondrial activity (MA), while in a deuterium-depleted medium an increase. This was established using mitotracker and rhodamine 123, and was also confirmed by expression of the UCP1 gene. The correlation dependence of mitochondrial activity in a medium with a changed ratio of deuterium/protium $(\mathrm{D} / \mathrm{H})$ and supramolecular structures was established, using the laser diffraction method. Density inhomogeneities in the deuterated medium are noted to be large, and in the deuterium-depleted medium small, in comparison with the control.
\end{abstract}

\section{Introduction}

Deuterium (D) is a non-radioactive stable isotope of hydrogen that is constantly present in natural water $(\mathrm{D} / \mathrm{H}=150 \mathrm{ppm})$ and in the human body (in amounts comparable to the contents of such essential trace elements as zinc, copper, iron et al.) [1, 2]. By replacing protium $(\mathrm{H})$ with deuterium in the molecule, deuterated compounds are obtained that are identical in size and structure. But due to the higher strength of carbon-deuterium (C-D) chemical bonds compared with carbon-protium $(\mathrm{C}-\mathrm{H})$, the properties of deuterated compounds change, for example, the reaction rates with their participation are reduced [3]. Recently, significant experimental material has been accumulated on the effect of the deuterium/protium $(\mathrm{D} / \mathrm{H})$ ratio in water on processes involving biologically active substances in vitro and in vivo [4-14]. It was noted that the kinetics of biological reactions depends on the ratio of deuterium/protium $(\mathrm{D} / \mathrm{H})$ in aqueous solutions. It was found that in

* Corresponding author: zlatskiy@ukr.net 
the range of deuterium content below natural concentrations $(<150 \mathrm{ppm}$, deuterium depleted water) (ddw), the kinetic isotope effect (KIE) of biologically active compounds increases [6, 15]. In such medium, the rates of dissolution of substances [16] and mutarotation of carbohydrates [17], the optical properties of chiral compounds [10, 15] change, the absorption and accumulation of ions of essential trace elements in medicinal and food plants increase [18]. Proven reduction in obesity in laboratory animals [19]. The use of ddw in oncology as an adjuvant is accompanied by an improvement in the condition of patients $[13,14]$.

However, the exact mechanism for changing the rate of biological reactions by changing the ratio of $\mathrm{D} / \mathrm{H}$ has not been established.

Mitochondrial activity (MA) is one of the markers of culture aging process. Therefore, we used the MA as an indicator of changes in the properties of the cancer stem cells lines and normal cultures in vitro, including cell aging during cultivating in medium with different isotopic composition.

The aim of our investigation was to study the mitochondrial activity of normal and cancer cells in vitro cultured in medium with various concentrations of deuterium.

\section{Materials and methods}

\subsection{Physicochemical analysis}

\subsubsection{Physicochemical analysis of water with different deuterium content}

The following basic water samples with different deuterium content were used in this study: deuterium-depleted water (ddw) with $\mathrm{D} / \mathrm{H}=4 \pm 2 \mathrm{ppm}$; $\mathrm{D} 2 \mathrm{O}$ - water with $\mathrm{D} / \mathrm{H}=99$ absolute at. \% D (all Sigma-Aldrich, USA). Growth media with various deuterium content were prepared by diluting deuterium-depleted and deuterated water. The following growth media were used in this study: No. 1 (ddw medium) - medium with D/H ratio 35 ppm; No. 2 (D2O medium) - the medium with the highest deuterium content - D/H ratio $500.000 \mathrm{ppm}$. The Milli-Q water served as a standard (Control medium) with D/H ratio $150 \mathrm{ppm}$. Milli-Q, deuterium-depleted and deuterated water had no differences in physical characteristics [5] or in trace element composition, except the deuterium content. This excluded the multifactor influence in the system for all comparison groups. The deuterium content was controlled by multipass laser absorption spectroscopy on the Isotopic Water Analyzer-9120032 (Los Gatos Research Inc., USA) [1, 5]. pH, redox potential (RP), electrical conductivity (EC), salinity were controlled with 8200 pH/ORP/Conductivity/TDS/Salt/temp.Meter (EZODO, Taiwan).

Chemical analysis of water with different deuterium content was performed by inductively coupled plasma-mass spectrometry on the ICP-QMS Agilent 7500CE spectrometer (Agilent Technologies, USA) [1]. The concentration of all elements in the milliQ, deuterium-depleted or deuterated water did not exceed the upper detection limit (detection limit range $-0.1-10 \mathrm{ppm}$ ). Detailed description of the methods was presented in previous studies $[1,5]$.

\subsubsection{Laser diffraction method for assessing cell culture metabolic activity}

Particle size analysis (numerical and volume distribution of particle size/dimension spectra), dissolution kinetics of powders of different dispersity were recorded by the method of Low-angle laser light scattering (LALLS) on diffraction particle analysers [23] using Master Sizer 2000 instrument, Zeta Sizer Nano ZS instrument (MALVERN 
Instruments, UK) and "Cluster-1" - IDL-1, laser meter of dispersion (manufactured by the Institute of Colloid Chemistry and Chemistry of Water, Ukraine; modification RUDN, Russia). Detailed description of the methods was presented in previous studies $[5,16]$.

\subsection{Cultivation of MS and cancer cells in vitro}

In experiment, we used dry standard substances (powder) for cells cultivation, which were dissolved in water samples with a modified isotopic composition.

\subsubsection{Cultivation of adipose derived stem cells in vitro}

The experiments with human cell cultures were carried out in accordance with the human experiment issues of the Code of Ethics of the World Medical Association (Declaration of Helsinki). In all cases, donors signed the voluntary informed consents.

We studied adipose-derived mesenchymal stem cells (ADSCs) during adipogenic differentiation. The cells were cultured using standard protocols. Detailed description of the methods was presented in previous study [20]. ADSCs were isolated from the lipoaspirate. The lipoaspirate were incubated in the $0.1 \%$ collagenase IA and $0.1 \%$ pronase with the addition of $2 \%$ FBS in modified MEM- $\alpha$ (all from Sigma-Aldrich, USA) for 2 hours at $+37^{\circ} \mathrm{C}$. The resulting cell suspension was washed twice with PBS (Sigma-Aldrich, USA) and was transferred to $25 \mathrm{~cm}^{2}$ cell culture flask (SPL, Korea). Cells were cultured in the following control growth medium: modified MEM- $\alpha$ (Sigma-Aldrich, USA) prepared from the powder by dilution with MilliQ water of natural isotope content supplemented with $10 \%$ FBS, $2 \mathrm{mM}$ L-glutamine, $100 \mathrm{U} / \mathrm{ml}$ penicillin, $100 \mu \mathrm{g} / \mathrm{ml}$ streptomycin and $1 \mathrm{ng} / \mathrm{ml}$ FGF-2 (all from Sigma-Aldrich, USA). Experimental growth media had a composition similar to the control one, but were prepared on the basis of deuterated and deuteriumdepleted waters. The cells were cultured in multi-gas incubator CB210 (Binder, Germany) at $+37{ }^{\circ} \mathrm{C}$ in the atmosphere of saturated humidity, $5 \% \mathrm{CO}_{2}$ and $5 \% \mathrm{O}_{2}$.

Cells were seeded with a density of 1000 cells per $1 \mathrm{~cm}^{2}$. In $24 \mathrm{~h}$ the control growth medium was changed for the experimental one.

Detailed description of the adipogenic differentiation was presented in previous study [21]. To determine the effect of deuterium adipogenic differentiation on the ADSCs biological properties, cell cultures from five different donors were assessed after 14 days of culturing in experimental media.

\subsubsection{Cultivation of cancer cell lines in vitro}

We studied cancer lines: SKOV3, U87, HT29, MCF7, JURCAT, ZR-75-1, mouse macrophages, mouse $\beta$-cells. Cancer lines were obtained from the American Type Culture Collection (ATSS). Detailed description of the methods was presented in previous study [1]. Cancer cell lines were cultured in DMEM medium prepared from the powder by dilution in the ddw, deuterated or Milli-Q water and sterilized by filtering. The culture media were additionally supplemented with $10 \%$ FBS, $2 \mathrm{mM}$ glutamine (all from SigmaAldrich, USA). The cells were cultured in $\mathrm{CO}_{2}$ incubators CB210 (Binder, Germany) at $+37{ }^{\circ} \mathrm{C}$ in the atmosphere of absolute humidity and $5 \% \mathrm{CO}_{2}$.

Cell lines were seeded in 24 well or 6 well plates in a density to reach $90 \%$ confluence in 24 hours. Afterwards the cells were cultured in serum free medium (to inhibit cell proliferation). Then the cells were washed three times and the culture medium was completely changed for the experimental medium for each group.

To determine the effect of deuterium on the cell lines biological properties, three cell lines at $\mathrm{P} 2$ were assessed, after 6 days of culturing in experimental media. 


\subsection{The MSC mitochondrial activity (Mitotraker)}

MA analysis was performed using a fluorescent dye Mitotraker (Thermo Fisher, USA) in ADSCs on a spectrofluorimeter (Labsystems Multiskan PLUS, USA). All evaluation procedures of MA were performed according to the standard protocol and according to the recommendations of the dyes manufacturer.

\subsection{The cancer cells mitochondrial activity (Rhodamine 123)}

MA analysis was performed using a fluorescent dye Rhodamine 123 (Thermo Fisher, USA) in cancer cell lines on a spectrofluorimeter (Labsystems Multiskan PLUS, USA). All evaluation procedures of MA were performed according to the standard protocol and according to the recommendations of the dyes manufacturer.

\subsection{The RT-qPCR assay}

Separately, adipogenic differentiation was performed by RT-qPCR analysis of UCP1 gene expression. Detailed description of the method was presented in previous studies [21]. Total RNA was isolated from ADSCs using NucleoZOL (MACHEREY-NAGEL GmbH \& Co. $\mathrm{KG}$, Germany) according to manufacturer's protocol. The RNA quality and concentration were determined with a spectrophotometer NanoDrop ${ }^{\text {TM }} 1000$ (Thermo Scientific, USA). 2 $\mu \mathrm{g}$ of isolated RNA were reverse transcribed to cDNA using RevertAid First Strand cDNA Synthesis kit (Thermo Scientific, USA). RT-qPCR was performed with a 7500 Real-Time PCR System (Applied Biosystems, CA, USA) using $5 \times$ HOT FIREPol ${ }^{\circledR}$ EvaGreen ${ }^{\circledR}$ qPCR Mix Plus (ROX) (Solis BioDyne, Estonia). The Applied Biosystems 7500 system software (V. 1.3.1) was used for data analysis. The primers sequences are listed in Table 1. The following PCR cycling conditions were applied: 10 min at $95^{\circ} \mathrm{C}, 40$ cycles of $10 \mathrm{~s}$ at $95^{\circ} \mathrm{C}$, and $40 \mathrm{~min}$ at $60{ }^{\circ} \mathrm{C}$. Expression level of TATA-box binding protein (TBP) was used as internal control. $\mathrm{Ct}$ values for target genes were normalized against $\mathrm{Ct}$ value of TBP at the same threshold level. The relative quantification (comparative $\mathrm{Ct}(\Delta \Delta \mathrm{Ct})$ method) was used to compare the expression levels of the target genes with the internal control. Dissociation curve analysis was performed after every run to check the primers specificity. Results were presented in relative units. For all conditions, reaction was performed three times (each gene in triplicate). GraphPad Prism 4 (GraphPad Software, USA) and MS Excel were used for statistical analysis and graphic data presentation.

\subsection{Statistics}

The data were reported as mean \pm SD for each group. Statistical analyses were performed using one-way analysis of variance (ANOVA) in Origin Pro software. Differences were considered to be statistically significant when $\mathrm{p}<0.05$.

\section{Results and discussion}

\subsection{Physics and chemical parameters and particle size analysis for assessing the cell culture in growth media with different deuterium content}

Using the laser diffraction method, we have found essential differences in structural inhomogeneities in culture media with different $\mathrm{D} / \mathrm{H}$ ratios (Fig. 1-3). This suggests that 
various groups of molecular and supramolecular structures were formed in the experimental systems.
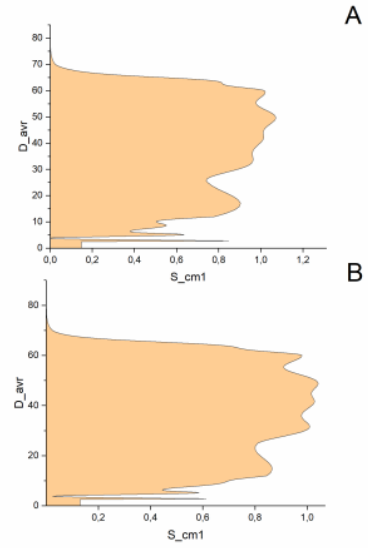
B

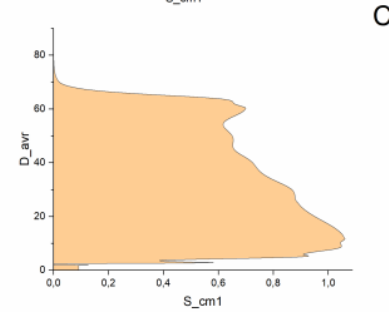

Fig. 1. Control medium before (A), 7 days (B), 14 days (C) cultivation
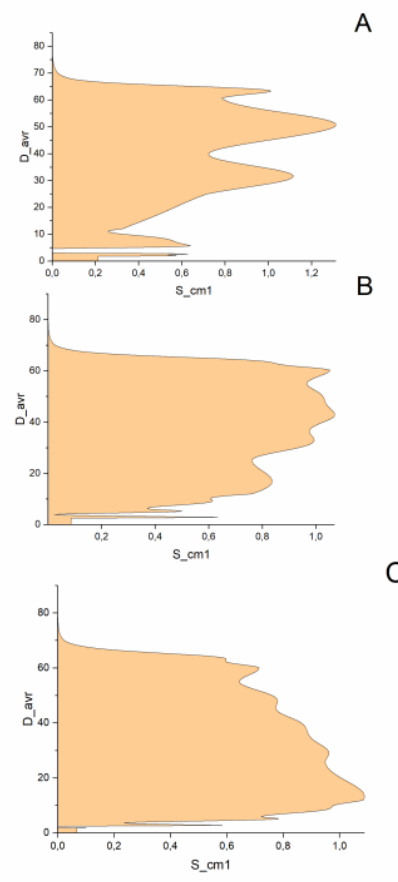

Fig. 2. D2O medium before (A), 7 days (B), 14 days (C) cultivation 

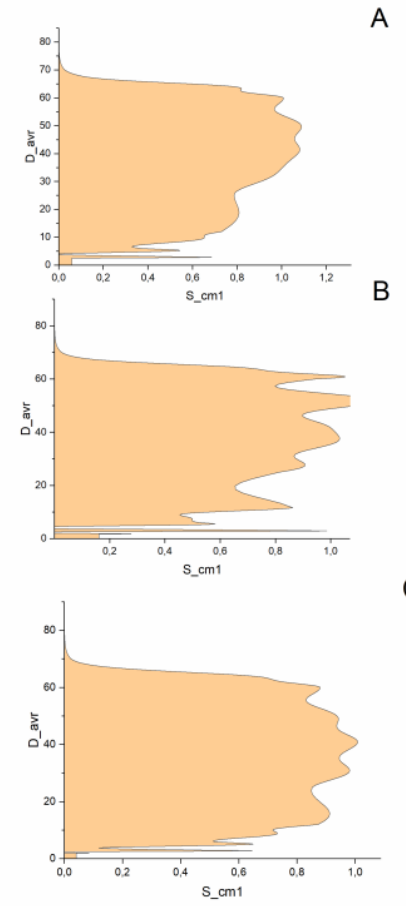

C

Fig. 3. ddw medium before (A), 7 days (B), 14 days (C) cultivation

Before cultivation, the smallest size and, accordingly, the largest number of optical inhomogeneities were observed in the control sample. It should be noted that the $\mathrm{D}_{2} \mathrm{O}$ medium was slightly different from the control medium. Ddw medium was characterized by larger size and, accordingly, a smaller number of optical inhomogeneities (Fig. 1-3 (A)).

After cultivation, the following tendency was observed: a decrease in the deuterium content in a sample lead to a noticeable increase in the size of optical inhomogeneities and, accordingly, to a decrease in their number. In this case, there was a multidirectional influence of the deuterium content compared to the corresponding medium before cultivation. So, there was a slight increase in the size of optical inhomogeneities in high deuterium concentrations $\left(\mathrm{D}_{2} \mathrm{O}\right.$ medium), and, on the contrary, a decrease in a sample with low deuterium content (Fig. 2, A). The reverse trend is observed for the number of optical inhomogeneities (Fig. 3, B). In the control medium changes before and after cultivation was not observed.

Changes in structural optical inhomogeneities (Fig. 1-3) can be explained by several factors. The deuterium/protium ratio can directly change the supramolecular structure of the medium, without influencing intracellular metabolism, as was noted earlier for the water with low salt composition $[1,5,11]$. However, it is likely that different $\mathrm{D} / \mathrm{H}$ ratio may cause different effects associated with the cell culture metabolic activity. Probably, in the studied media, cells secrete metabolites of a different nature, respectively, various structural heterogeneities are formed, which are detected using laser diffraction. Thus, changes in inhomogeneities in culture media could be the result of changes in intracellular metabolism caused by different $\mathrm{D} / \mathrm{H}$ ratios.

Considering the different metabolism of cells in culture media with different $\mathrm{D} / \mathrm{H}$ ratios, we decided to compare different groups for our experimental system during adipogenic differentiation. 


\subsection{Mitochondrial activity and UCP1 gene expression in culture ADSCs growth media with different deuterium content}

The levels of the study MA in the Control group were set at $100 \%$, and changes in their concentrations are given as the percentage change relative to the Control group.

Mitotracker dyes are fluorescent markers of MA. In our study, using Mitotracker dye showed (Fig. 4) that the most intense MA was observed in in vitro ADSCs cultured in ddw medium. Growth medium with a high concentration of deuterium $\left(\mathrm{D}_{2} \mathrm{O}\right)$ inhibited MA.

Previously [21], it was shown that during adipogenic differentiation in ADSCs, brown fat is formed in the ddw, and white fat in the deuterated medium. Therefore, we used the indicator expression of the UCP1 gene. UCP1 is an uncoupling protein found in the mitochondria of beige adipose tissue (BAT) [22, 23]. In our study, showed (Fig. 4) that the most intense expression $U C P 1$ was observed in ADSCs cultured in ddw medium. On the other hand, deuterated growth medium $\left(\mathrm{D}_{2} \mathrm{O}\right)$ inhibited expression $U C P 1$.

Taking into account the obtained data of the Mitotracker dye and expression of the $U C P 1$ gene, the main mechanism of action of high/low concentrations of deuterium should be sought in the work of the respiratory chain and the mitochondrial complex itself.

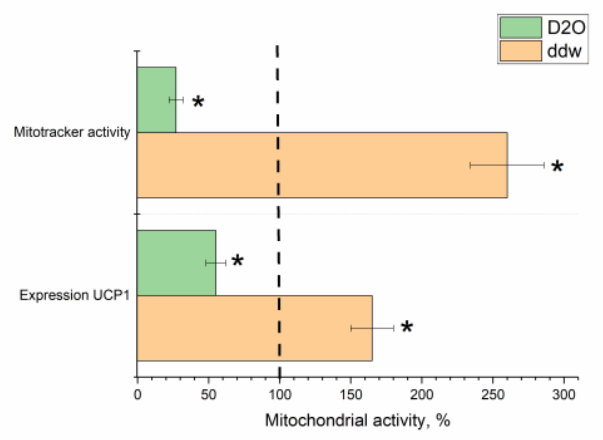

Fig. 4. In vitro MA (Mitotracker activity and UCP1 expression) diagram of ADSCs in growth medium with different deuterium content. (Mean $\pm \mathrm{SD} n=6, *-\mathrm{p}<0.01$ comparison with the control group). Control - marked with a dotted line.

\subsection{Mitochondrial activity cancer cells in growth media with different deuterium content}

It is known that the fluorescence intensity of rhodamine 123 labelling is directly correlated with the mitochondria activity in viable cells [24]. This indicator is often used to evaluate the viability of cancer cells. In our opinion, there are no conflict in the use of different markers in determining MA. Because the Mitotracker is a derivative of rhodamine, has a similar structure and properties. However, at the same time, the Mitotracker is a more sensitive dye for working with MMSC. Since there are few studies with Mitotracker and cancer lines, we used the classic MA label for cancer cells - rhodamine 123.

Our study showed (Fig. 5) that the most intense MA is observed in cancer cell lines in vitro cultured in ddw medium. Growth medium with a high concentration of deuterium (D2O) inhibited MA in cancer cell lines in vitro. The levels of the study MA in the Control group were set at $100 \%$, and changes in their concentrations are given as the percentage change relative to the Control group. 


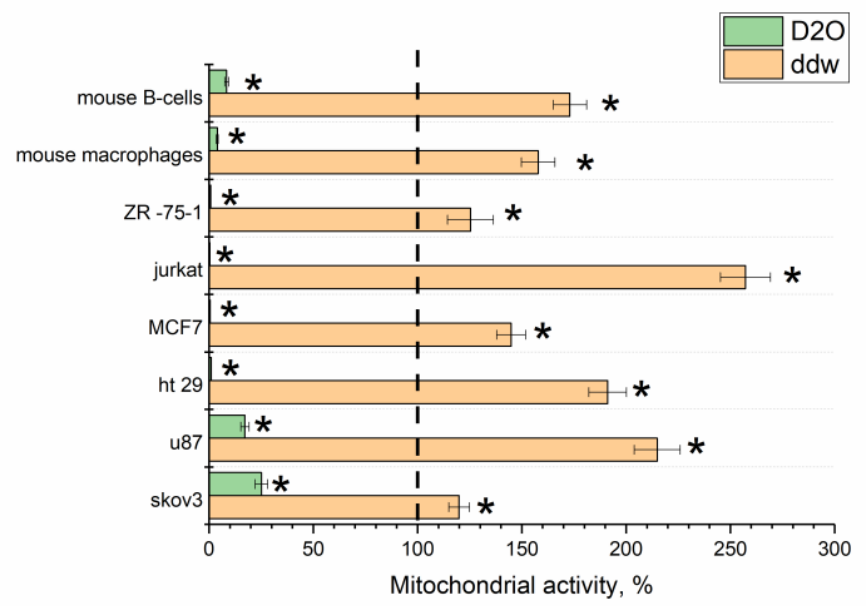

Fig. 5. In vitro MA (Rhodamine 123) diagram of cancer cells in culture medium with different deuterium content. (Mean $\pm \mathrm{SD} \mathrm{n}=6, *-\mathrm{p}<0.01$ comparison with the control group). Control marked with a dotted line.

The data obtained confirm that the possible mechanism of the action of different concentrations of deuterium on the reaction rate must be sought in the activity of mitochondria. It seems that the mechanism of action of deuterium is similar, both for normal cell lines and for cancerous cultures.

In addition, a change in density inhomogeneities in culture media indicates a different metabolism under conditions of different ratios of deuterium/protium (Fig. 1-3). The results of MA (Fig. 4-5) and, accordingly, the possible different pathways of metabolism in cells cultured in medium with different concentrations of deuterium confirm this.

The obtained data indicate significant effects of aging in a cell culture, which was cultivated in medium with a high deuterium content. On the other hand, a slowdown in the effects of aging were observed during stem cells culturing in a deuterium-depleted environment. In addition, UCP1 gene expression data partially reveal MA mechanisms at different deuterium / protium ratios.

\section{Conclusion}

The study showed (Fig. 1) that the most intense MA was observed in normal and cancer cell lines in vitro cultured in ddw medium. Growth medium with a high concentration of deuterium $\left(\mathrm{D}_{2} \mathrm{O}\right)$ inhibited MA. It is established that the inhomogeneities of densities in the deuterated medium are large and that in the deuterium-depleted medium they are small compared to the control.

Further research in the field of isotopic control of aging cell cultures in vitro should be directed to the study of the exact mechanisms and signalling pathways. In general, this will allow using a personalized approach in biology and medicine to the diagnosis and treatment of aging effects.

\section{Acknowledgments}

The publication has been prepared with the support of the "RUDN University Program 5-100". 


\section{References}

1. A. Syroeshkin, et al, J. Trace Elem. Med. Biol., 50, 629-633 (2018)

2. M. Zoroddu, et. al., Journal of Inorganic Biochemistry (2019) doi:10.1016/j.jinorgbio.2019.03.013.

3. T. Pirali, et al, Journal of Medicinal Chemistry, 62(11), 5276-5297 (2019) doi:10.1021/acs.jmedchem.8b01808

4. R. Tung, Future Med. Chem., 8(5), 491-494 (2016)

5. A. Syroeshkin, et al, Chemical Engineering Journal, 377, 119827 (2019) doi:10.1016/j.cej.2018.08.213

6. V. Goncharuk, et al, J. of Water Chem. and Technol., 39(2), 97-102 (2017)

7. O. Levitskaya et al, Pharmaceutical Chemistry Journal, 49(11), 779-781 (2016)

8. O. Titorovich, et al, Pharmaceutical Chemistry Journal, 48(12), 842-844 (2015)

9. E. Tsisanova, et al, Trace elements in medicine, 11(2), 8 (2010)

10. A. Syroeshkin, et al, IJAP, 10(5), 20182 (2018)

11. S. Dzhimak, et al, Life Science, 6(2), 1 (2016)

12. V. Goncharuk, at al, Journal of Water Chemistry and Technology, 33(1), 15-25 (2011)

13. G. Somlyai, et al, Biomacromol J., 2(1), 1-7 (2016)

14. K. Krempels, et al, J Cancer Res Ther., 1(8), 194-200 (2013)

15. V. Goncharuk, et al, Journal of Water Chemistry and Technology, 39(6), 319-324 (2017)

16. E. Uspenskaya et al, Indian Journal of Pharmaceutical Sciences, 80(2), 318-324 (2018)

17. O. Zrelov, et al, Pharmaceutical Chemistry Journal, 49(6), 413-416 (2015)

18. E. Harvey, Biol Bull., 66(2), 91-96 (1934)

19. T. Halenova, et al, Molecules, 25, 23 (2020) doi:10.3390/molecules 25010023

20. A. Zlatska, et al, Sci. World J., 5454367, (2018) doi:10.1155/2018/5454367

21. A. Zlatska, et al, Sci Rep., 10, 5217 (2020) doi:10.1038/s41598-020-61983-3

22. P. Crichton, et al, Biochimie., 134, 35-50 (2017) doi:10.1016/j.biochi.2016.12.016

23. L. Zhao, et al, Structure, 25(9), 1371-1379.e3. (2017) doi:10.1016/j.str.2017.07.005

24. A. Baracca et al, Biochimica et Biophysica Acta , 1606, 137- 146 (2003) 